\title{
Reactive power operability of distributed energy resources for voltage stability of distribution networks
}

\author{
Shuai HU ${ }^{1}$, Yue XIANG ${ }^{1}$, , Xin ZHANG $^{2}$, Junyong LIU ${ }^{1}$, \\ Rui WANG ${ }^{1,3}$, Bowen HONG ${ }^{4}$
}

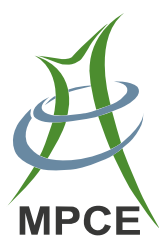

\begin{abstract}
The penetration level of distributed energy resources (DERs) is increasing and has significant impact on the voltage stability of distribution networks. Based on the various types of DERs with distinct reactive power characteristics (RPC), their different contributions to the system voltage stability require classification. Firstly, the features of DERs are reviewed and classified based on their RPC, to investigate different distributed generation technologies for reactive power support in distribution networks. Then, the concept of a relative available
\end{abstract}

CrossCheck date: 18 October 2018

Received: 18 April 2018/Accepted: 18 October 2018/Published online: 3 January 2019

(C) The Author(s) 2019

$\bowtie$ Yue XIANG

xiang@scu.edu.cn

Shuai HU

shuai.scu@foxmail.com

Xin ZHANG

xin.sam.zhang@gmail.com

Junyong LIU

liujy@scu.edu.cn

Rui WANG

Elaine1126@outlook.com

Bowen HONG

bowenhh@sina.com

1 College of Electrical Engineering and Information Technology, Sichuan University, Chengdu 610065, China

2 Electricity National Control Centre, National Grid, Wokingham RG41 5BN, UK

3 State Grid Shizuishan Power Supply Company, Shizuishan 753000, China

4 State Grid Energy Research Institute, Beijing 102209, China transmission capacity index (RATCI), which is based on power transfer margin of the power-voltage curve considering the non-negligible distribution network resistance, is proposed to quantify and evaluate the voltage stability by integrating DERs with the defined reactive power types. Case studies have been conducted for an IEEE 33-bus distribution network to calculate the system RATCI for the mixed integration of DERs. Results show that the multitype and multi-locational integration of DERs can improve the voltage stability of a distribution network.

Keywords Voltage stability, Distributed energy resources, Distribution networks, Relative available transmission capacity, Reactive power operability, Renewable energy integration

\section{Introduction}

Static voltage stability analysis is considered as one of the primary tools for transmission capacity assessment, which guarantees sufficient voltage stability margin for the safety and reliability of the power supply in distribution networks. However, the decentralized and decarbonized energy future leads to the shortfall of energy supply to meet the demand, the conflicts between economic development and environmental protection, and the unbalance between resource location and load distribution [1]. The electricity industry has paid increasing attention to the distributed energy resources (DERs) and used them to address and overcome these challenges. Typically, DERs are connected to the middle-low voltage distribution networks and serve as the power supply for local demand [2, 3].

Generally, the voltage stability of a distribution network needs to be maintained within certain operational limits, in 
order to avoid unacceptable voltage deviations on networks assets and customer connection points. As a result, the planning of reactive power compensation devices is a critical concern for improving the system resilience to voltage instability, enhancing the power transfer capacity and ensuring the power quality [4]. Hence, the research on conventional reactive power compensation devices to support system voltage stability has been widely conducted. In [5], based on the $N-1$ outage risk index of each transmission line, the candidate lines for the static var compensator (SVC) configuration are determined. The modal analysis is then implemented to search the most sensitive line to voltage stability and determine the SVC installation. Moreover, in [6], an improved particle swarm optimization algorithm is proposed to specify and optimize (rather than maximize) the voltage stability margin for combined optimal sitting and sizing of static synchronous compensator (STATCOM) in the transmission networks. In [7], the distribution network optimal planning was combined with the STATCOM optimal configuration to improve the reliability and operating efficiency of the distribution networks. However, the integration of DERs provides an alternative option for reactive power control in distribution networks. In [8], several distribution network optimal planning methods, by considering stochastic behaviors of DERs, were reviewed. In the review, the DERs are deemed to provide active power to meet grid demand, but their inherent and potential reactive power support capabilities need to be studied.

The optimal sitting and sizing of DERs are two major concerns when planning DERs for voltage stability of distribution networks. In [9], the most sensitive bus to voltage collapse was selected as the priority location to place the DER for the voltage stability and power transfer capacity improvement. Then, the group of candidate buses were further analyzed by modal analysis in critical situations to decide the final installation locations of the DERs [10]. In [11], the load variations and voltage stability margin enhancements were considered to study their correlations with DER optimal planning in distribution networks, with an improved imperialistic competitive algorithm to solve the proposed model under each loading level. These papers have studied the reactive power support ability of a specific DER type, with no considerations of various DER types and their distinct reactive power contributions to distribution network operations. The existing classifications of DER types are demonstrated in [12-14]. Based on the generation technology, they are classified into photovoltaic, wind-turbine, fuel cell, micro-gas turbine, distributed small hydropower, etc. Based on the grid interface technology, they are classified into asynchronous interface, synchronous interface and power electronic interfaces. Based on the adopted bus types in a power flow calculation, they are classified into PV bus, PQ bus, PI bus and $\mathrm{PQ}(\mathrm{V})$ bus. However, for voltage stability in a distribution network, classifications based on the reactive power characteristics (RPC) of each DER type should be considered.

Besides the allocation of DERs in distribution networks, the assessment method is another key point for analyzing the improvement in static voltage stability. Existing static voltage stability index include: sensitivity index, singular value/eigenvalue index, Thevenin equivalent based index, second order index and margin index, which are primarily applied to the transmission network assessment [15]. For distribution network planning, in [16], a voltage stability index was proposed to show the sensitivity of each bus to voltage collapse. In [17], a voltage stability margin index was presented to determine the DER optimal placement and evaluate the voltage stability improvement. However, the voltage phase angle difference between the original bus and terminal bus was ignored. In [18], a simplified indicator based on the Thevenin model and voltage measurement was proposed to estimate the voltage stability margin and transmission capacity. These papers proved that the voltage stability margin index is a useful assessment tool for static voltage stability. Such indices can effectively reflect the relative margin between the initial operating and critical points of the voltage collapse.

In order to search the critical point of the system, there are primarily three methods: direct method, nonlinear programming method and continuous method. Briefly, the direct method has relatively low computational accuracy due to its approximation approach. The nonlinear programming method has heavily computational difficulties due to the number of constraints [19]. Thus, the continuous method is adopted in this paper. The continuation power flow (CPF) method is a competent tool for static voltage stability analysis, which has been widely studied since it was developed in [20]. Subsequent studies have been conducted to improve the CPF method, which are summarized here as the continuous parameter selection [21], parameterization method [22] and step size control [23] et al. However, the above CPF methods are primarily applied in transmission networks. Therefore, several literatures have introduced the conventional CPF method into the distribution networks. A continuation three-phase distribution network power flow method considering the regulation of transformers was presented in [24]. For the meshed distribution networks, a fast convergence loopanalysis-based power flow (LBPF) method was analyzed in [25]. Based on previous studies, a novel LBPF-based CPF was developed to be a voltage stability analysis tool for both radial and meshed distribution networks. In [26], system state variables were partly eliminated and the reduced power flow equations were combined with 
conventional $\mathrm{CPF}$ to find the maximum loading margin of the distribution networks. In [27], due to the larger ratio of $R / X$ in distribution networks, two sets of recursive system equations were presented to improve the calculation efficiency as no Jacobin matrix was required.

The main contributions of this paper are: (1) classifying the reactive power types of DERs and the ability for voltage regulation; (2) developing a novel index, relative available transmission capacity index (RATCI), to evaluate the impact of the reactive power types of DERs on the voltage stability in distribution networks; (3) studying the single-type and multi-type integration of DERs in various scenarios considering the reactive power type, integration location, total capacity and capacity allocation; (4) recommending strategies for optimal mixed integration of DERs to achieve maximum voltage stability or maximum DER penetration levels.

The rest of this paper is structured as follows. Section 2 presents the novel classification of DERs based on RPC. Section 3 proposes a RATCI method to assess the voltage stability of distribution networks with DERs. In Section 4, the single-type and multi-type mixed integration of various types of DERs are studied in an IEEE 33-bus distribution network. Conclusions are drawn in Section 5.

\section{Classification of DERs based on RPC}

\subsection{Definition of reactive power categories for DERs}

DERs are power generation systems that inject active power into the system. However, DERs' reactive power generation and absorption abilities are very different based on their RPC. This paper proposes a novel classification method of DERs based on their RPC into the following four reactive power categories:

1) Type P-RQ: DERs in this category only dispatch active power, with either no reactive power support or non-participation in the system voltage regulation.

2) Type P-IQ: DERs in this category can simultaneously inject active power and reactive power into the system.

3) Type P-CQ: DERs in this category are not only an active power source, but also a reactive load. Their operation requires them to consume reactive power from the system.

4) Type P-V-Q: DERs in this category can work towards a target voltage by either generating or absorbing reactive power. Their flexibility in reactive power dispatch makes this type as the potential voltage regulating sources in the system.

\subsection{Review and mapping DERs into four reactive power categories}

In this section, five common DG techniques: photovoltaic, wind turbine, fuel cell, micro turbine and distributed small hydropower are reviewed and mapped into four reactive power types as follows.

\section{1) Photovoltaic}

Photovoltaic system generates DC electricity and convert it to $\mathrm{AC}$ through an inverter. The existing photovoltaic inverter can be classified as a voltage-source inverter (VSI) and current-source invertor (CSI) on the DC side. For grid connected inverters, their respective control techniques can be classified as the voltage-control mode and current-control mode on the AC side. As this paper will focus on voltage stability on system levels, the voltage-control and current-control of the photovoltaic inverter which have direct impact on the grid side will be studied.

The current-control mode of the photovoltaic inverter directly controls the phase and amplitude of the current, which is able to withstand the interference of the grid voltage fluctuation as the primary control mode [28]. This mode can keep the output current in-phase with the grid voltage by tracking grid voltage, i.e., operating in the unity power factor. Due to its non-participation in voltage regulation, the photovoltaics with a current-control mode inverter are recommended to be the Type P-RQ.

However, the voltage-control mode of the photovoltaic inverter not only requires the synchronization of the output voltage with system voltage, but also uses the output voltage to adjust the output current. This mode allows the inverter to support local voltage in distribution networks by injecting reactive power into the grid. The reactive power can be controlled by altering the $q$-axis current. Therefore, the photovoltaic with the voltage-control mode inverter is classified as Type P-IQ.

\section{2) Wind turbine}

Wind turbine generation schemes include the constant speed constant frequency systems (CSCF) and the variable speed constant frequency systems (VSCF).

The CSCF wind turbine generator systems directly connect to the grid system. Theoretically, either a synchronous or asynchronous generator is able to achieve wind energy conversion to electricity. Nevertheless, the synchronous generator based CSCF wind-turbine system is rarely used, due to several disadvantages in practical applications such as a complicated structure involving power converters, low energy conversion efficiency, poor overload capacity and high cost, etc. Generally, an asynchronous (induction) generator is widely used in CSCF wind turbine systems. It is necessary to convert the low 
rotation speed of the wind turbine to the high speed of the rotor by mechanical transmission equipment, and then drive the shaft of the asynchronous generator to produce electric power to the grid system $[29,30]$.

The active power $P$ of the CSCF wind-turbine can be considered as constant, along with the terminal voltage $V_{\mathrm{G}}$, which is determined by the grid. Thus, the reactive power $Q$ is formulated as:

$Q=-\left(\frac{V_{\mathrm{G}}^{2}}{X_{\mathrm{m}}}+\frac{V_{\mathrm{G}}^{2}-\sqrt{V_{\mathrm{G}}^{4}-4 P^{2} X_{\delta}^{2}}}{2 X_{\delta}}\right)$

where $X_{\mathrm{m}}$ is the excitation reactance; $X_{\delta}$ is the sum of the stator leakage reactance and the rotor leakage resistance.

Thus, the CSCF wind turbine systems consume reactive power to be Type P-CQ.

The VSCF wind-turbine systems include the directlydriven permanent magnet synchronous generator (PMSG) or the AC-excited doubly fed induction generator (DFIG), both are converter-based systems. Owing to the decoupling of the active and reactive power control, VSCF wind-turbine systems have the ability to operate at variable speed and flexibility in a reactive power dispatch. Thus, the VSCF wind turbine systems belong to Type P-V-Q.

\section{3) Fuel cell}

The fuel cell (FC) is connected to the grid system via a power conditioning unit (PCU). The equivalent circuit is shown in Fig. 1.

As shown in Fig. 1, $V_{\mathrm{FC}}$ is the DC voltage of the FC, $\dot{V}_{\text {AC }}$ is the rms of the PCU output voltage, $X_{\text {eq }}$ is the output equivalent reactance, $\dot{V}_{\mathrm{S}}$ is the system voltage, $m$ is the modulation depth of PCU, $\Psi$ is the advance angle of PCU [31]. The active and reactive power to the grid system can be expressed as follows.

$$
\left\{\begin{array}{l}
P=\frac{\left|\dot{V}_{\mathrm{AC}}\right|\left|\dot{V}_{\mathrm{S}}\right|}{X} \sin \Psi \\
Q=\frac{\left|\dot{V}_{\mathrm{AC}}\right|\left|\dot{V}_{\mathrm{S}}\right|}{X} \cos \Psi-\frac{\left|\dot{V}_{\mathrm{AC}}\right|^{2}}{X}
\end{array}\right.
$$

where $\left|\dot{V}_{\mathrm{AC}}\right|=m V_{\mathrm{FC}}$.

From the above formula, the control of the FC's active and reactive power output can be performed by regulating $m$ and $\Psi$ of the PCU respectively. Hence, FC is recommended to be Type P-IQ.

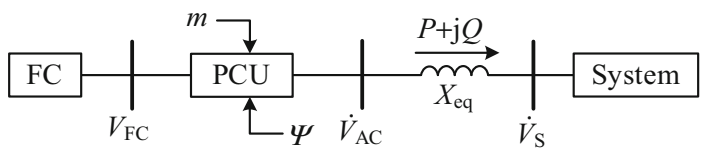

Fig. 1 Grid-connection equivalent circuit of FC

\section{4) Micro turbine}

A micro turbine (MT) operates at high speed. It requires a frequency converter if it directly drives the PMSG, or via a speed regulation gearbox to the PMSG.

Generally, if the MT that operates at a constant power factor excitation control mode does not consume reactive power from the grid system, it belongs to Type P-IQ. The MT with PMSG on the grid side could operate at constant voltage excitation control mode, which is able to maintain the bus voltage by controlling the reactive power output, thus it is classified as Type P-V-Q.

\section{5) Distributed small hydropower}

Distributed small hydropower (DSH) generation systems connect to the grid through a synchronous generator, similar to the conventional hydropower plant. The main differences are more flexible construction and less impact on the ecological system. In addition, DSH's capacity is much smaller, and normally lower than $1 \mathrm{MW}$. Practically, synchronous generators in DSH rarely operate in an underexcitation mode, but mostly in an over-excitation mode with a high-power factor. Therefore, the excitation control is generally in a constant power factor control mode [32], which means the DSH will simultaneously inject active and reactive power into the system. It is therefore recommended to be Type P-IQ.

Table 1 summarizes the different generation technologies of DERs in four reactive power categories: Type P-RQ, Type P-IQ, Type P-CQ and Type P-V-Q.

\section{RATCI assessment}

In this section, based on the power-voltage curve for voltage stability analysis, a RATCI is proposed to quantify the system's voltage stability margin with the integration of DERs, which considers the non-negligible resistance in power distribution networks. For demonstration purposes, the RATCI is derived mathematically in a simplified single machine infinite bus power system, with generator terminal voltage $\dot{E}$, infinite bus voltage $\dot{V}$, transmission line impedance $\dot{Z}$, active power $P$ and reactive power $Q$. The proposed RATCI assessment method is shown in Fig. 2.

First, the complex power $\dot{S}$ delivered to the infinite bus can be obtained by:

$$
\begin{aligned}
\dot{S} & =\dot{V} \dot{I}^{*}=\dot{V}\left(\frac{\dot{E}-\dot{V}}{R+\mathrm{j} X}\right)^{*} \\
& =\frac{1}{R^{2}+X^{2}} \dot{V}(R+\mathrm{j} X)\left(\dot{E}^{*}-\dot{V}^{*}\right) \\
& =\frac{1}{R^{2}+X^{2}}(R+\mathrm{j} X)\left(E V \cos \theta+\mathrm{j} E V \sin \theta-V^{2}\right)
\end{aligned}
$$


Table 1 Typical representations of DERs in four reactive power categories

\begin{tabular}{ll}
\hline Type & Typical representatives \\
\hline P-RQ & Photovoltaic with current-control inverter \\
P-IQ & Photovoltaic with voltage-control inverter, MT with frequency converter, FC, \\
& DHS, MT with synchronous generator (constant power factor excitation control) \\
P-CQ & CSCF wind turbines \\
P-V-Q & VSCF wind turbines, MT with synchronous generator (constant voltage excitation control)
\end{tabular}

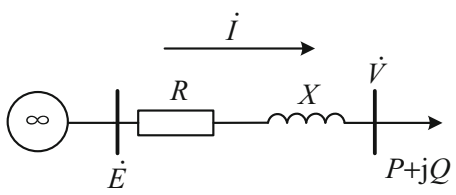

Fig. 2 Equivalent circuit diagram of typical single machine infinite bus system

where $\theta$ is the angle difference between $\dot{V}$ and $\dot{E}$; * represents the conjugate function.

By separating the active power and reactive power from the complex power, (3) is rewritten as:

$\left\{\begin{array}{l}P=\frac{1}{R^{2}+X^{2}}\left(R E V \cos \theta-X E V \sin \theta-R V^{2}\right) \\ Q=\frac{1}{R^{2}+X^{2}}\left(X E V \cos \theta+R E V \sin \theta-X V^{2}\right)\end{array}\right.$

Then, by multiplying $P$ and $Q$ with $R$ and $X$ respectively, (4) can be modified to:

$\left\{\begin{array}{l}R Q-X P=E V \sin \theta \\ R P+X Q=E V \cos \theta-V^{2}\end{array}\right.$

Eliminating $\theta$ of (5), the equation is shown as:

$V^{4}+\left(2 R P+2 X Q-E^{2}\right) V^{2}+\left(R^{2}+X^{2}\right)\left(Q^{2}+P^{2}\right)=0$

The discriminant of (6) is:

$\Delta=\left(2 R P+2 X Q-E^{2}\right)^{2}-4\left(R^{2}+X^{2}\right)\left(Q^{2}+P^{2}\right)$

To interpret (7) in the power-voltage curve, when the discriminant $\Delta>0$, the system operates $P_{0}$ at the left side of the critical point, i.e. $P_{0}<P_{\text {cri }}$, which means that the system voltage is stable. When the discriminant $\Delta=0$, the system operates at the critical point, i.e. $P_{0}=P_{\text {cri }}$, which means that the power flow has reached the maximum transmission capacity for system voltage stability; when the discriminant $\Delta<0$, the power flow has infeasible solutions, which means the voltage collapse has happened. In order to find the critical point $P_{\text {cri }}$, we make $\Delta=0$ and incorporate $Q=P \tan \phi$ :
$(2 R \tan \phi-2 X)^{2} P^{2}+4 E^{2}(R+X \tan \phi) P-E^{4}=0$

where $\phi$ is the power factor angle of the load.

By solving (8), we have obtained the solution for $P_{\text {cri }}$ :

$P_{\text {cri }}=\frac{E^{2}}{2} \cdot \frac{-(R \tan \phi+X) \pm|Z| / \cos \phi}{(X-R \tan \phi)^{2}}$

For the two roots of $P_{\text {cri }}$, the positive $P_{\text {cri }}$ is the maximum power flow to reach the critical point of the static voltage stability. Thus, the RATCI of the system at a given initial point $\left(P_{0}, \phi_{0}\right)$, is defined as shown in (10).

$$
\begin{aligned}
R A T C I & =\left(P_{\text {cri }}-P_{0}\right) / P_{\text {cri }} \\
& =1-\frac{2 P_{0}}{E^{2}} \cdot \frac{\left(X-R \tan \phi_{0}\right)^{2}}{|\dot{Z}| / \cos \phi_{0}-\left(R+X \tan \phi_{0}\right)}
\end{aligned}
$$

The proposed RATCI provides a quantitative index to show the voltage stability margin at each bus. The higher the RATCI value, the more stable is the voltage as the initial point is further away from the critical voltage collapse point.

\section{Case study}

\subsection{Case description}

In this paper, an IEEE 33-bus distribution network is selected for the case study, the system information and detailed parameters can be obtained in [12]. A distribution substation is connected at bus 1 . The CPF method is used to analyze the system voltage stability. To calculate RATCI for the entire distribution network, the load at each bus is scaled up proportionally until a voltage collapse is found. The type and size of each DER are given in Table 2.

The voltage security constraint is set within $\pm 7 \%$ of the nominal voltage. It should be noted that the initial voltage conditions of the 33-bus distribution network, before integrating any DERs, are unsatisfied by the voltage security constraint. This demonstrates a weak distribution network that requires reactive power support. 
Table 2 Basic generation capacity of DERs in each reactive category

\begin{tabular}{llll}
\hline Type & $\begin{array}{l}\text { Active } \\
\text { power }(\mathrm{kW})\end{array}$ & $\begin{array}{l}\text { Reactive } \\
\text { power }(\mathrm{kvar})\end{array}$ & $\begin{array}{l}\text { Voltage } \\
\text { (p.u.) }\end{array}$ \\
\hline P-RQ & +500 & - & - \\
P-IQ & +500 & +300 & - \\
P-CQ & +500 & -300 & - \\
P-V-Q & +500 & $-300 \sim+150$ & 1.0 \\
\hline
\end{tabular}

The impact of DERs on the system voltage stability is primarily dependent on the type, size and location. Therefore, it is necessary to comprehensively analyze and discuss the various types of DER that are integrated on the multiple locations with different capacities.

\subsection{Single-type integration of DERs}

The four types of DERs (P-RQ, P-IQ, P-CQ and P-V-Q) with capacity ranging from $0.5 \mathrm{MW}$ to $4 \mathrm{MW}$ are integrated into each single bus, respectively. The system's RATCI in each scenario are calculated. Since there are many different scenarios showing similar patterns of the RATCI variation, the typical bus in each type is chosen and drawn in Fig. 3 for the following analysis. The RATCI value of the basic network is 0.6885 , which is regarded as the benchmark for other DER integration scenarios. Specifically, the RATCIs for the scenarios which dissatisfy the voltage security constraint are reflected as the "flat points" in Fig. 3.

In general, after the integration of a certain capacity of DER into a bus, the system voltage starts regulating within the voltage security constraint. At the same time, the increase in system RATCI has been observed, indicating more robustness in the system voltage stability. Therefore, it is doubtless that the integration of the DER will improve the system RATCI and voltage stability.

More specifically, as shown in Fig. 3a, with Type P-RQ integration, the RATCI of the distribution network keeps rising followed by the increase in DER capacity, as well as the system voltage. It may exceed the upper limit of the voltage security constraint when reaching a certain DER capacity (the maximum RATCI 0.7744 is achieved by integrating at bus 13 with $3.5 \mathrm{MW}$ ). Taking bus 18 as an example, the maximum RATCI appears at the DER size of 2 MW and then the over-limit voltage happens as the DER capacity continues to rise which makes the RATCI system flat again. The increase in RATCI is due to the active power support from the integrated DERs to supply the local load, reduce the power flow in distribution network, and thus increase the voltage and "relative margin" in the power-voltage curve. In summary, results show that even

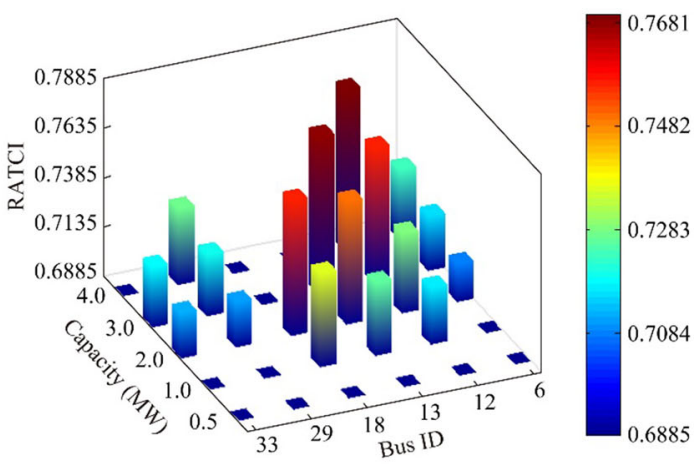

(a) Type P-RQ

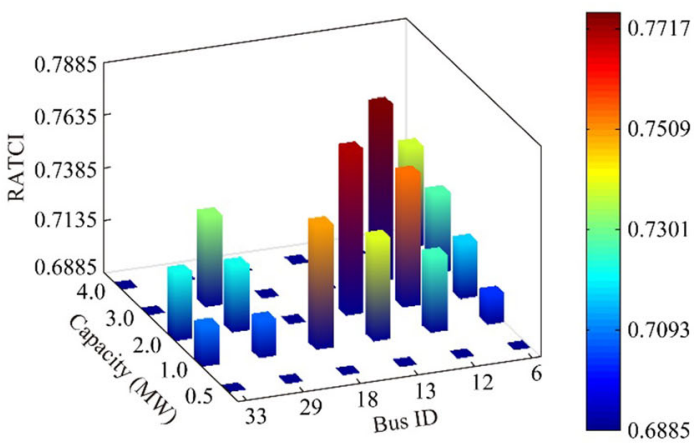

(b) Type P-IQ

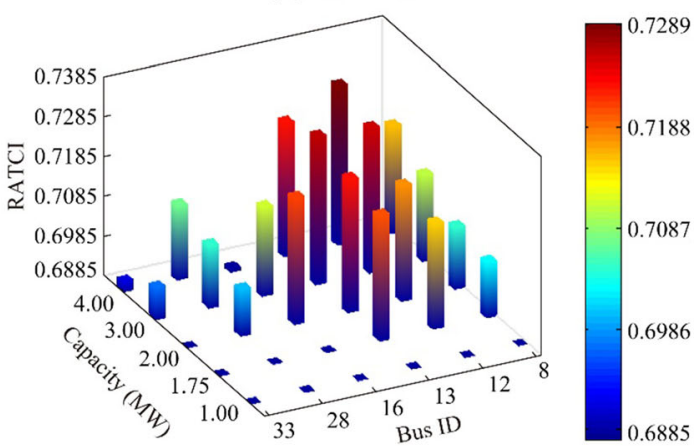

(c) Type P-CQ

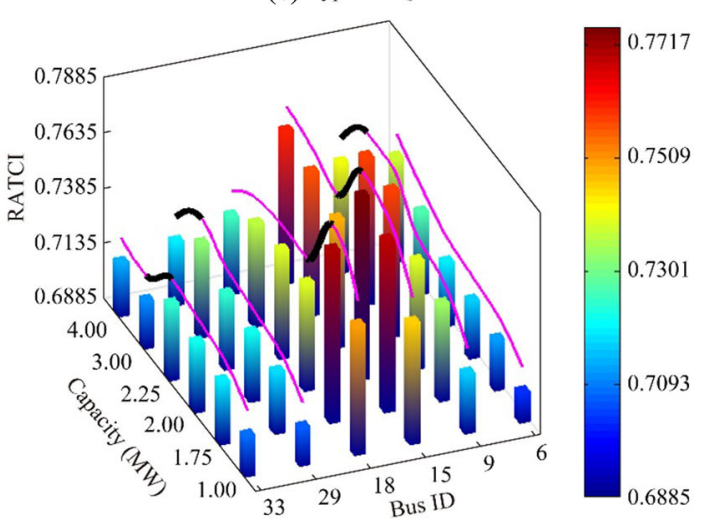

(d) Type P-V-Q

Fig. 3 RATCI for single-type integration of four types of DERs

with no reactive power support from Type P-RQ, DERs are still able to contribute voltage stability for the distribution networks. 
Table 3 Multi-type DERs allocation plans when TC of DERs is fixed

\begin{tabular}{llll}
\hline Scenario & TC $(\mathrm{MW})$ & DER location, type and capacity allocation & RATCI \\
\hline 1 & 0.5 & $\left\{I I_{12}, I_{18}, I V_{29}, I I_{33}\right\} @[10 \%, 30 \%, 40 \%, 20 \%]$ & 0.7052 \\
2 & 0.5 & $\left\{I I I_{12}, I_{18}, I V_{29}, I I_{33}\right\} @[10 \%, 40 \%, 10 \%, 40 \%]$ & 0.7075 \\
3 & 0.5 & $\left\{I I_{12}, I V_{18}, O_{29}, I V_{33}\right\} @[16.7 \%, 50 \%, 0 \%, 33.3 \%]$ & 0.7158 \\
4 & 0.5 & $\left\{O_{12}, I I_{18}, O_{29}, I I_{33}\right\} @[0 \%, 87.5 \%, 0 \%, 12.5 \%]$ & 0.7227 \\
\hline
\end{tabular}

Table 4 Multi-type DERs allocation plans when achieving target RATCI

\begin{tabular}{llll}
\hline Scenario & TC $(\mathrm{MW})$ & DER location, type and capacity allocation & RATCI \\
\hline 1 & 1 & $\left\{O_{12}, I_{18}, I_{29}, I I_{33}\right\} @[0 \%, 66.6 \%, 16.7 \%, 16.7 \%]$ & 0.73 \\
2 & 2 & $\left\{I_{12}, I_{18}, I I_{29}, I_{33}\right\} @[20 \%, 10 \%, 30 \%, 40 \%]$ & 0.73 \\
3 & 2.5 & $\left\{I V_{12}, O_{8}, I I_{29}, O_{33}\right\} @[33.3 \%, 0 \%, 66.7 \%, 0 \%]$ & 0.73 \\
4 & 3 & $\left\{I I_{12}, O_{18}, I I I_{29}, I I_{33}\right\} @[12.5 \%, 0 \%, 50,37.5 \%]$ & 0.73 \\
\hline
\end{tabular}

In Fig. 3b, Type P-IQ is more effective than Type P-RQ for voltage support. Taking buses 6, 29 and 33 as examples, the system RATCI starts increasing from $1 \mathrm{MW}$ Type P-IQ, whereas for the same bus, the system requires higher capacity of Type P-RQ for RATCI improvement. Such differences are due to the additional reactive power injection from Type P-IQ into the system, which helps to boost system voltage profiles and shift the "initial point" further away from the "critical point" in the power-voltage curve, so that RATCI can be effectively increased. The disadvantage of Type P-IQ is the excessive reactive power injection may happen if the DER capacity is over-sized (max RATCI 0.7747 is attained by integrating at bus 13 with $2.375 \mathrm{MW}$ ). One example can be taken from bus 18; the system cannot cope with $2 \mathrm{MW}$ of Type P-IQ due to the voltage exceeding the upper limit, whereas $2 \mathrm{MW}$ of Type P-RQ can be accommodated to achieve the best possible RATCI value.

By comparing results from Fig. 3a, b, it is found that the integration location of DERs makes a significant difference in system RATCI and the associated voltage stability. Bus 6 which is electrically closed to the transmission network, receives a steady increase in system RATCI following the capacity expansion for both Type P-RQ and Type P-IQ. However, buses 12, 13 and 18 show a faster rate of increase in RATCI and exhibit the higher risks of voltage violation to the upper limit. The reason is that the buses which are closed to the transmission network will get reactive support from the main interconnected system, so that this part of the distribution networks is "strong" enough to minimize the voltage deviation. In contrast, the distribution network which is remote from the main transmission system is "brittle" and subject to a bigger voltage deviation with DER integration. Therefore, the DER capacity needs to be carefully planned by considering the locational impact in the distribution networks. From this study, the large size of Type P-RQ and Type P-IQ are recommended to be connected closely to the main interconnected network, while the remote parts of the distribution networks are more suitable for the smaller size of the DERs but will receive higher effectiveness in voltage stability improvement.

In Fig. 3c, the DERs with Type P-CQ consume reactive power which would further depress the voltage profiles of the distribution networks. However, this type of DER also injects active power into the local demand areas to support the voltage stability. The two contradictory effects will either bring benefits or disadvantages in voltage stability with integrating a certain DER capacity, dependent on the integration locations (the maximum RATCI 0.7288 is achieved by integrating at bus 12 with $4 \mathrm{MW}$ ). For example, the terminal buses 18 and 33 have zero or low RATCI values regardless of DER capacity, their associated static voltages are always below the lower-limit of the voltage security constraint, which indicates that none or little benefits have been received from Type P-CQ for voltage support. Therefore, it is not recommended to integrate Type P-CQ to the terminal buses of distribution networks. When the integration moves towards the middle of the distribution networks, such as with buses 12, 13 and 16, higher RATCI values are observed, which indicates that voltage stability improvement is being received for these locations. When the DERs are closer to the original bus coupled with the transmission network, the drops in RATCI values have indicated lesser benefits in voltage regulation. In summary, the middle areas of the distribution network are ideal to integrate Type P-CQ of DERs for maximum voltage stability improvement.

In Fig. 3d, Type P-V-Q of DERs can provide both voltage regulation and reactive support, and are suitable to almost every bus for a wide range of integration capacity 
Table 5 Multi-type DERs allocation plans when TC of DERs is fixed while achieving target RATCI

\begin{tabular}{llll}
\hline Scenario & TC $(\mathrm{MW})$ & DER location, type and capacity allocation & RATCI \\
\hline 1 & 1 & $\left\{O_{12}, I_{18}, I I I_{29}, O_{3}\right\} @[0 \%, 50 \%, 50 \%, 0 \%]$ & 0.7204 \\
2 & 1 & $\left\{I_{12}, I I_{18}, I V_{29}, I_{33}\right\} @[70 \%, 10 \%, 10 \%, 10 \%]$ & 0.7204 \\
3 & 1 & $\left\{I I_{12}, I I_{18}, I I I_{29}, I I I_{33}\right\} @[10 \%, 30 \%, 40 \%, 20 \%]$ & 0.7204 \\
4 & 2 & $\left\{I_{12}, O_{18}, O_{29}, I I I_{33}\right\} @[87.5 \%, 0 \%, 0 \%, 12.5 \%]$ & 0.7501 \\
5 & 2 & $\left\{I V_{12}, I_{18}, I I_{29}, I_{33}\right\} @[20 \%, 30 \%, 40 \%, 10 \%]$ & 0.7501 \\
\hline
\end{tabular}

from $1 \mathrm{MW}$ to $4 \mathrm{MW}$. Particularly the over-sized DER capacity problems existing in the previous three Types are solved by Type P-V-Q (max RATCI 0.7741 is attained by integrating at bus 15 with $2.125 \mathrm{MW}$ ). For example, when integrating DER to bus 13 , the system has over voltage problems if DER capacity reaches $4 \mathrm{MW}$ with the Type P-RQ, Type P-IQ and Type P-CQ, but retains sufficient RATCI margin for the Type P-V-Q integration. The reason is that Type $\mathrm{P}-\mathrm{V}-\mathrm{Q}$ is able to provide reactive power absorption ability to regulate high voltage issues, therefore providing additional support for voltage stability. Due to the flexibility in reactive power dispatch, Type P-V-Q of DERs could improve voltage stability for almost every part of the distribution network and support its own DER capacity expansion if required.

Since the DERs with Type P-V-Q are flexible for integration into wide areas of the distribution network, it is worth finding the optimal locations for the different sizes of Type P-V-Q. A novel "cliff point" is defined to describe the maximum RATCI amount of the different DER capacities for a fixed bus, which are shown as black line segments in Fig. 3d. For example, the system "cliff point" of 0.7669 is achieved by integrating the $1.75 \mathrm{MW}$ Type P-V-Q into bus 18 . For a larger capacity of $2 \mathrm{MW}$, the "cliff point" appears at the location of bus 15 . When the DER's capacity reaches $3 \mathrm{MW}$, the "cliff point" of RATCI will shift to the bus 9. The results indicate that the larger the Type P-V-Q size, the closer to the original bus the DER is required to be. This phenomenon can be explained by the fact that the larger size of the DER not only requires support from its own P-V-Q capability, but also from the distribution network. DER integration locations that are close to original bus are likely to receive more robust reactive power support from the main system.

\subsection{Multi-type integration of DERs}

In previous sections, comprehensive work has been done to discuss the optimal locations for DERs with different reactive types. In this section, by screening out the groups of buses which have similar effectiveness on system voltage stability, the four-distinguished buses, noted as buses
12, 18, 29 and 33, are chosen as the typical locations for DER integration. Then, the corresponding system RATCI is calculated by integrating DERs with various combinations of total capacity (TC), capacity allocation and reactive power type.

In order to represent the DER scenarios for multi-type integration, the following format is adopted:

$$
\begin{aligned}
R A T C I= & \left\{T_{1}, T_{2}, \cdots, T_{n}\right\} @\left[P_{1}, P_{2}, \cdots, P_{n}\right] \\
& T_{1}, T_{2}, \cdots, T_{n} \in\{0, I, I I, I I I, I V\}
\end{aligned}
$$

where $T_{n}$ is the installed DER type at buses $n ; 0, I, I I, I I I, I V$ represent None, Type P-RQ, Type P-IQ, Type P-CQ and Type P-V-Q respectively; $P_{n}$ is the percentage of TC that is allocated to bus $n$, and $P_{1}+P_{2}+\ldots+P_{n}=100 \%$.

Then, various DER integration scenarios are studied with the associated RATCI values found in Tables 3-6.

First, with the fixed DER capacity, the multi-type integration and single-type integration are compared. As shown in Table 3, previously with single-type integration of $0.5 \mathrm{MW}$ DERs, the system voltage always violates the static security constraint regardless of DER type. However, under the total capacity of $0.5 \mathrm{MW}$, a multi-type integration with all 4 types of DERs enhances the RATCI value to 0.7052. Other multi-type integration scenarios also show the RATCI improvement compared to single-type integration. Therefore, the overall results prove that multi-type integration with various DER types is more effective than single-type integration in regulating system voltage.

In Table 3, the first two scenarios are built with the same DER types, but different percentages of capacity allocation. Scenario \#2 allocates more capacities to terminal buses 18 and 33, which subsequently improves the RATCI value by 0.0023 . Other scenarios show similar RATCI improvement by either integrating more supportive Type P-V-Q (Scenario \#3), or even allocating all DER capacities to terminal buses 18 and 33 (Scenario \#4). The remote locations of the distribution network are more likely to breach the voltage security constraint and drag down the system RATCI. Therefore, more DER capacity allocation is required for remote locations so that the overall system RATCI can be effectively improved. 
Table 6 Multi-type DERs allocation plans when aims to achieve optimal TC of DERs or RATCI while other one is fixed

\begin{tabular}{llll}
\hline Scenario & TC $(\mathrm{MW})$ & DER location, type and capacity allocation & RATCI \\
\hline 1 & 2 & $\left\{I I_{12}, I_{18}, I I I_{29}, I_{33}\right\} @[20 \%, 10 \%, 30 \%, 40 \%]$ & 0.73 \\
2 & 2 & $\left\{I V_{12}, I_{18}, I I_{29}, I_{33}\right\} @[20 \%, 30 \%, 40 \%, 10 \%]$ & 0.7501 \\
3 & 2 & $\left\{O_{12}, I V_{18}, O_{29}, I I_{33}\right\} @[0 \%, 75 \%, 0 \%, 25 \%]$ & 0.7745 \\
4 & 0.5 & $\left\{I I I_{12}, I_{18}, I V_{29}, I I_{33}\right\} @[10 \%, 30 \%, 40 \%, 20 \%]$ & 0.7 \\
5 & 2.5 & $\left\{O_{12}, O_{18}, I I I_{29}, I I I_{33}\right\} @[0 \%, 0 \%, 25 \%, 75 \%]$ & 0.7 \\
\hline
\end{tabular}

In Table 4, the scenarios that use different TC to achieve the same RATCI are illustrated. To minimize the required TC of the DERs for the reduction in investment cost, the priority selection types of DERs are Type P-RQ, Type P-IQ and Type P-V-Q. These three types offer effective voltage support capabilities as discussed previously. In contrast, if the maximum $\mathrm{TC}$ is set as the objective to allow higher DER penetration levels, then Type P-CQ becomes useful for consuming the excessive reactive power from other DER Types, and to balance the reactive power for system voltage stability. When the TC requirement increases, more capacity is recommended to allocate to Type P-CQ for upward voltage regulation.

In Table 5, by tuning the DER's type and capacity allocation, the same RATCI with identical TC can be achieved for several DER integration scenarios. These results show that when distribution network planners allocate type and capacity of DERs with the targets of fixed TC and RATCI, there are multiple integration scenarios available to choose. Practical scenarios are dependent on the geographical limitation, energy resource distribution, local policy and customer agreement. Other economic factors such as capital investment cost of different types of DERs are also considered. In general, the cost of Type P-RQ and Type P-CQ is cheaper than Type P-IQ and Type P-V-Q. Overall, if bus 12 and bus 33 are unsuitable to build DERs due to the environmental, political or economic limitations as indicated above, Scenario \#1 could be chosen as the suitable DER integration strategy.

In Table 6 , when the TC is fixed at $2 \mathrm{MW}$, the maximum RATCI is set as the objective with the enumeration method to find the optimal solution. It is determined that scenario \#3 has the highest RATCI value of 0.7745 . As discussed previously, the most supportive DERs of Type P-V-Q and P-IQ are selected at the terminal bus to achieve the maximum possible RATCI. When the RATCI is fixed at 0.7 , the maximum DER penetration rate (total capacity) is set as the objective. The enumeration method found that Scenario \#5 has the maximum TC of 2.5 MW. The strategy used in this scenario is to connect DERs of Type P-CQ that can consume excessive reactive power from the system, in order to regulate high voltage issues caused by DER penetration.

\section{Conclusion}

This paper classifies the DERs into four reactive power categories: Type P-RQ, Type P-IQ, Type P-CQ and Type P-V-Q, based on their RPC. Then, a novel RATCI, which is based on a power-voltage curve considering the non-negligible distribution network resistance, is proposed to evaluate the voltage stability of the distribution network with the integration of DERs. Using an IEEE 33-bus distribution network, studies are conducted to calculate the RATCI for various DER integration scenarios including single-integration and multi-integration of the DER's reactive power type, location, total capacity and capacity allocation. Conclusions are drawn as follows.

1) Type P-CQ of the DER is particularly useful for solving high voltage issues and improving the upward voltage security margin of the distribution networks. It is recommended to use Type P-CQ in conjunction with Type P-IQ and Type P-RQ, to balance the reactive power for system RATCI improvement.

2) The integration location of the DER makes a significant difference in the voltage stability of distribution networks. The terminal buses remote from the main interconnected system are "brittle" and subject to high voltage deviation. Therefore, the terminal regions in the distribution networks are ideal locations for Type P-V-Q of the DER to regulate system voltage.

3) In order to achieve the highest penetration rate of the DERs while still maintaining voltage security, DERs with Type P-CQ are recommended to be used to consume excessive reactive power due to the higher penetration of other DER types.

In future studies, the proposed RATCI method and RPCbased reactive type classification can be further conducted to develop an optimization method for the mixed integration and configuration of multi-type DERs, with the possible optimization objectives of maximum RATCI level and DER penetration rate. In addition, the capital investment cost for each DER type will be established, together with the RATCI method to minimize the DER planning and investment cost in distribution networks. 
Acknowledgements This work was supported by National Natural Science Foundation of China (No. 51807127), the Fundamental Research Funds for the Central Universities of China (YJ201654), and the Open Research Subject of Key Laboratory of Sichuan Power Electronics Energy-saving Technology and Devices (szjj2017-052).

Open Access This article is distributed under the terms of the Creative Commons Attribution 4.0 International License (http:// creativecommons.org/licenses/by/4.0/), which permits unrestricted use, distribution, and reproduction in any medium, provided you give appropriate credit to the original author(s) and the source, provide a link to the Creative Commons license, and indicate if changes were made.

\section{References}

[1] Katiraei F, Iravani MR (2006) Power management strategies for a microgrid with multiple distributed generation units. IEEE Trans Power Syst 21(4):1821-1831

[2] Abri RSA, El-Saadany EF, Atwa YM (2013) Optimal placement and sizing method to improve the voltage stability margin in a distribution system using distributed generation. IEEE Trans Power Syst 28(1):326-334

[3] Wang L, Gao H, Zou G (2017) Modeling methodology and fault simulation of distribution networks integrated with inverterbased DG. Protec Contr Mode Power Syst 2(1):31

[4] Kamarposhti MA, Lesani H (2011) Effects of STATCOM, TCSC, SSSC and UPFC on static voltage stability. Electr Eng 93(1):33-42

[5] Chang YC (2012) Multi-objective optimal SVC installation for power system loading margin improvement. IEEE Trans Power Syst 27(2):984-992

[6] Rocha L, Castro R, Jesus JMF (2016) An improved particle swarm optimization algorithm for optimal placement and sizing of STATCOM. Int Trans Electr Energy 26(4):825-840

[7] Kumar D, Samantaray SR (2016) Implementation of multi-objective seeker-optimization-algorithm for optimal planning of primary distribution systems including DSTATCOM. Int J Electr Power 77:439-449

[8] Xiang Y, Liu J, Li F et al (2016) Optimal active distribution network planning: a review. Elect Power Comp Syst 44(10):1075-1094

[9] Hedayati H, Nabaviniaki SA, Akbarimajd A (2008) A method for placement of DG units in distribution networks. IEEE Trans Power Del 23(3):1620-1628

[10] Ettehadi M, Ghasemi H, Vaez-Zadeh S (2013) Voltage stabilitybased DG placement in distribution networks. IEEE Trans Power Del 28(1):171-178

[11] Poornazaryan B, Karimyan P, Gharehpetian GB et al (2016) Optimal allocation and sizing of DG units considering voltage stability, losses and load variations. Int J Electr Power 79:42-52

[12] El-Khattam W, Salama MMA (2004) Distributed generation technologies, definitions and benefits. Electr Power Syst Res 71(2):119-128

[13] Prakash P, Khatod DK (2016) Optimal sizing and siting techniques for distributed generation in distribution systems: a review. Renew Sust Energ Rev 57:111-130

[14] Balamurugan K, Srinivasan D (2012) Review of power flow studies on distribution network with distributed generation. In: Proceedings of IEEE 9th international conference on power electronics and drive systems, Singapore, 5-8 December 2012, $7 \mathrm{pp}$
[15] Modarresi J, Gholipour E, Khodabakhshian A (2016) A comprehensive review of the voltage stability indices. Renew Sust Energ Rev 63:1-12

[16] Chakravorty M, Das D (2001) Voltage stability analysis of radial distribution networks. Electr Power Energy Syst 23(2):129-135

[17] Murty VVSN, Kumar A (2015) Optimal placement of DG in radial distribution systems based on new voltage stability index under load growth. Int J Electr Power 69:246-256

[18] Pérez-Londoño S, Rodríguez LF, Olivar G (2014) A simplified voltage stability index (SVSI). Int J Electr Power 63:806-813

[19] Mansour MR, Alberto LFC, Ramos RA (2016) Preventive control design for voltage stability considering multiple critical contingencies. IEEE Trans Power Syst 31(2):1517-1525

[20] Ajjarapu V, Christy C (1992) The continuation power flow: a tool for steady state voltage stability analysis. IEEE Trans Power Syst 7(1):416-423

[21] Alves DA, Silva LCPD, Castro CA et al (2003) Alternative parameters for the continuation power flow method. Electr Power Syst Res 66(2):105-113

[22] Canizares CA, Alvarado FL (2002) Point of collapse and continuation methods for large AC/DC systems. IEEE Trans Power Syst 8(1):1-8

[23] Chen K, Hussein A, Bradley ME et al (2003) A performanceindex guided continuation method for fast computation of saddle-node bifurcation in power systems. IEEE Trans Power Syst 18(2):753-760

[24] Zhang XP (2006) Continuation power flow in distribution system analysis. In: Proceedings of IEEE PES power systems conference and exposition, Atlanta, USA, 29 October-1 November 2006, 5 pp

[25] Ju Y, Wu W, Zhang B et al (2014) Loop-analysis-based continuation power flow algorithm for distribution networks. IET Gener Transm Dis 8(7):1284-1292

[26] Dukpa A, Venkatesh B, El-Hawary M (2009) Application of continuation power flow method in radial distribution systems. Electr Power Syst Res 79(11):1503-1510

[27] Aly MM, Abdel-Akher M (2016) A robust quasi-direct continuous power-flow analysis using two-bus equivalents for voltage stability analysis of radial distribution systems. Electr Eng 100(1):47-57

[28] Mahmud MA, Pota HR, Hossain MJ (2014) Nonlinear current control scheme for a single-phase grid-connected photovoltaic system. IEEE Trans Sustain Energ 5(1):218-227

[29] Devbratta T, Nadarajah M (2009) Influence of constant speed wind turbine generator on power system oscillation. Electr Mach Power Syst 37(5):478-494

[30] Chen HY, Chen JF, Duan XZ (2006) Study on power flow calculation of distribution system with DGs. Auto Electr Power Syst 30(1):35-40

[31] Lasseter R (2001) Dynamic models for micro-turbines and fuel cells. In: Proceedings of power engineering society summer meeting, Vancouver, Canada, 15-19 July 2001, 6 pp

[32] Borkowski D, Węgiel T (2013) Small hydropower plant with integrated turbine-generators working at variable speed. IEEE Trans Energy Conver 28(2):452-459

Shuai HU received the B.S. degree in electrical engineering and automation from Sichaun Univesity. He is currently pursing the M.S. degree in power system and automation in Sichuan University, Chengdu, China. His research interests include distribution network planning and distributed energy resource.

Yue XIANG received the B.S. and Ph.D. degrees from Sichuan University, China, in 2010 and 2016, respectively. From 2013 to 
2014, he was a joint Ph.D. student at the Department of Electrical Engineering and Computer Science, University of Tennessee, Knoxville, US and also a visiting scholar at the Department of Electronic and Electrical Engineering, University of Bath, UK in 2015. Now he is an associate research professor in the College of Electrical Engineering and Information Technology, Sichuan University, China. His main research interests are electric vehicles, power system planning and optimal operation, renewable energy integration and smart grids.

Xin ZHANG received his B.Eng. degree in automation from Shandong University, China, in 2006; his M.S. and Ph.D. degrees in electrical power engineering from The University of Manchester, U.K., in 2007 and 2010 respectively. He is a senior power system engineer in the Electricity National Control Centre at the National Grid, U.K. He is currently responsible for national energy scheduling and transmission analysis of the GB power system, and previously worked in the areas of electricity network planning and market operation. His main research interests include power system planning and operation; smart distribution networks with the integration of renewable and multi-vector energy sources.

Junyong LIU received the Ph.D. degree from Brunel University, UK, in 1998. He is a professor in the School of Electrical Engineering and
Information, Sichuan University, China. His main research areas of interest are power market, power system planning, operation, stability and computer applications.

Rui WANG received the B.S. degree in electrical engineering and automation from Sichaun Univesity. She is currently pursing the M.S. degree in power system and automation in Sichuan University, Chengdu, China. She has been working in the Dept. of Operation and Maintanance of the State Grid Shizuishan Power Supply Company since 2017. Her research interests is power market.

Bowen HONG received his B.S. degree from Sichuan University, Chengdu, China, in 2008, and received his Ph.D. Degree from Tianjin University, Tianjin, China, in 2014, both in Electrical Engineering. He has been working in the Dept. of Renewable Energy of the State Grid Energy Research Institute Co. Ltd. since 2014. His research interests include operational and business modes of distributed generators, energy storage and micro-grid, and planning of active distribution network and integrated energy system. 